\title{
CONF $950905--46$ \\ SYSTEM CONTROL \\ AND DATA ACQUISITION \\ OF THE TWO NEW FWCD \\ RF SYSTEMS AT DIII-D
}

\author{
by \\ T.E. HARRIS, J.C. ALLEN, W.P. CARY, \\ S.W. FERGUSON,* C.C. PETTY, and R.I. PINSKER
}

This is a preprint of a paper presented at the 16th IEEE/NPSS Symposium on Fusion Engineering, September 30-October 5, 1995, Champaign, Illinois, and to be printed in the Proceedings.

Work supported by

U.S. Department of Energy

Contract DE-AC03-89ER51114

and $W-7405-E N G-48$

DESTREUMON OF THUS DOCUREENT IS UNUMUTED

*Lawrence Livermore National Laboratory

GENERAL ATOMICS PROJECT 3466

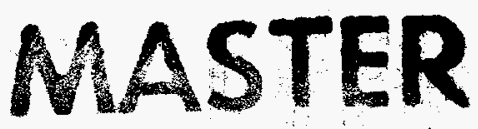
OCTOBER 1995 


\section{DISCLAMMER}

Portions of this document may be illegible in electronic image products. Images are produced from the best available original document. 


\section{DISCLAIMER}

This report was prepared as an account of work sponsored by an agency of the United States Government. Neither the United States Government nor any agency thereof, nor any of their employees, make any warranty, express or implied, or assumes any legal liability or responsibility for the accuracy, completeness, or usefulness of any information, apparatus, product, or process disclosed, or represents that its use would not infringe privately owned rights. Reference herein to any specific commercial product, process, or service by trade name, trademark, manufacturer, or otherwise does not necessarily constitute or imply its endorsement, recommendation, or favoring by the United States Government or any agency thereof. The views and opinions of authors expressed herein do not necessar. ily state or reflect those of the United States Government or any agency thereof. 


\title{
System Control and Data Acquisition of the Two New FWCD RF Systems at DIII-D*
}

\author{
T.E. Harris, ${ }^{a}$ J.C. Allen, ${ }^{a}$ W.P. Cary, ${ }^{a}$ S.W. Ferguson, ${ }^{b}$ C.C. Petty, ${ }^{a}$ and R.I. Pinsker ${ }^{a}$ \\ ${ }^{a}$ General Atomics, P.O. Box 85608, San Diego, California 92186-9784

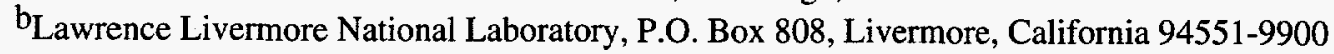

\begin{abstract}
The Fast Wave Current Drive (FWCD) system at DIII-D has increased its available radio frequency (RF) power capabilities with the addition of two new high power transmitters along with their associated transmission line systems. A Sun Sparc-10 workstation, functioning as the FWCD operator console, is being used to control transmitter operating parameters and transmission line tuning parameters, along with acquiring data and making data available for integration into the DIII-D data acquisition system. Labview, a graphical user interface application, is used to manage and control the above processes. This paper will discuss the three primary branches of the FWCD computer control system: transmitter control, transmission line tuning control, and FWCD data acquisition. The main control program developed uses VXI, GPIB, CAMAC, Serial, and Ethernet protocols to blend the three branches together into one cohesive system. The control of the transmitters utilizes VXI technology to communicate with the transmitter's digital interface. A GPIB network allows for communication with various instruments and CAMAC crate controllers. CAMAC crates are located at each phaseshifter/stub-tuner station and are used to digitize transmission line parameters along with transmission line fault detection during RF transmission. The phase-shifter/stub-tuner stations are located through out the DIII-D facility and are controlled from the FWCD operator console via the workstation's Serial port. The Sun workstation has an Ethernet connection allowing for the utilization of the DIII-D data acquisition "Open System" architecture and of course providing communication with the rest of the world.
\end{abstract}

\section{INTRODUCTION}

The Fast Wave Current Drive (FWCD) system at DIII-D has been upgraded with the addition of two new high power radio frequency (rf) transmitters along with their associated transmission line systems [1]. It was decided to have one central control console, running one main program, for operating and monitoring all the sub-system devices associated with the FWCD system. A Sun Sparc-10 workstation was chosen as the FWCD control console and LabView was chosen as the software used to develop the main control program [2]. There are three primary control branches in the main control program; the transmitter remote control, the transmission line tuning control, and the data acquisition control. These three branches use various intercommunication protocols to transfer information between hardware devices and software routines as displayed in Fig. 1. This paper will discuss the three primary branches of the main control program and their intercommunication protocols.

\section{TRANSMITTER REMOTE CONTROL}

The transmitter manufacturer made available a digital interface which allows the transfer of digital information representative of the transmitter state. It also provides a mode for remote control of the transmitter. The digital interface consists of a bank of relay $\mathrm{I} / \mathrm{O}$ and TTL logic devices. The control console uses VXI (VMEbus eXtensions for Instrumentation) technology to interface with the transmitter I/O devices via a MXI (Multi-system eXtension Interface) bus connected directly to the console's S-bus [3]. A Tektronix 4287 "Differential 32-Channel Analog/Digital Comparator" is used to receive information from the transmitter and a Tektronix 4353 "32-Ch. SPST 5A General Purpose Relay Switching" module is used to communicate digital information to the transmitter. The transmitter local control panel can be mimicked on the control console display by selecting the transmitter control loop in the main control program.

The transmitter mode of the main FWCD program was designed to perform two primary functions; transmitter control and transmitter parameter changes. When in the transmitter control window, the console operator can perform the transmitter start-up procedure. If an auxiliary system fails to come on-line, then the problem will be indicated on the console display and the proper action can be taken to remedy the problem. Parameter changes can be made in the transmitter change window. One of eleven pre-programmed frequency channels can be selected which will change the source frequency and initiate the inter-stage tuning adjustments between the three output amplifiers for the selected transmitter. Also, pulse width limits and output power leveling can be set. Unfortunately due to the manufacturers production deficiencies, we have been unable to test and implement our developed transmitter mode software.

\section{RF TRANSMISSION LINE TUNING CONTROL}

The rf transmission line connecting the rf transmitters to the DIII-D vessel consists of twenty tuning devices, such as

\footnotetext{
*Work supported by the U.S. Department of Energy under Contract Nos. DE-AC03-89ER51114 and W-7405-ENG-48.
} 


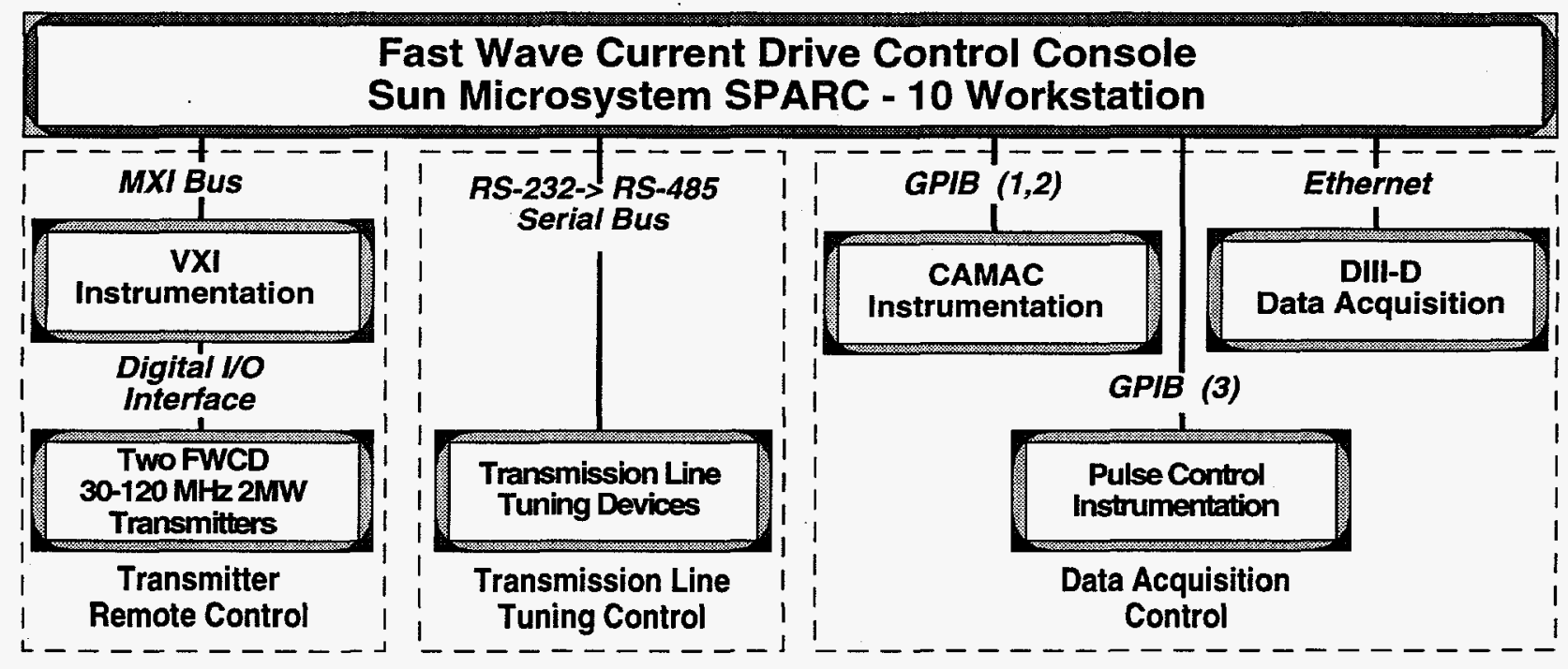

Fig. 1. FWCD inter-communication network.

phase shifters and stub tuners, that are quite large and require a servo-motor drive system for operation. These tuning elements are distributed throughout the DIII-D facility in areas which are off limits during DIII-D plasma operations; this makes local control of the servo system impossible for tuning the transmission line system. Each tuner element has a RS-485 serial interface for remote programming to move or read the status of the device. Therefore, the serial (RS-232) port on the main control console is used to communicate to all the tuning elements via an RS-232 $>$ RS-485 converter. Depending on the operating mode, the console operator can quickly make tuning changes to optimize if transmission line system performance. The operator calls up the Main Tuner window from the main program and selects the appropriate method for changing tuner positions; the auto mode, manual mode, or the quick tune option.

The auto mode is used primarily during DIII-D machine operation and uses ASCII file read/write calls for tuner position values. In this mode the FWCD console operator can reposition the tuners based on current DIII-D plasma parameters, a previous DIII-D shot setup, or to a vacuum conditioning setup. Based on plasma parameters, the physics operator located in the DIII-D control room can send programatically calculated tuning positions to an ASCII file on the FWCD control console located in the FWCD control room via an ethernet connection. The main control program reads these values and moves the appropriate tuning elements accordingly. If re-positioning based on previous shot positions is requested, then the console operator enters the preferred shot number and the tuners are re-positioned to the setup for the entered shot number. When vacuum conditioning is desired, the console operator chooses the vacuum position option and the tuners move to their respective positions for vacuum conditioning.

The manual mode is used to move a specific tuner or tuners to any position. A window is displayed on the console which displays all the tuners' current positions. The operator can then enter the new position values for the desired tuners and send the command which moves all the necessary tuners at the same time.

The quick tune mode is most useful during vacuum conditioning. While operating the transmitter at a specified repetition rate, the operator can adjust the phase shifters and stub tuners to properly match both transmission line systems to the DIII-D tokamak vacuum. As the tuners are moving, the transmission line positions can be monitored and the tuners can be stopped as soon as a proper match has been attained. Once the match has been attained, the tuner settings can be written to an ASCII type file which can be used by either the auto-mode or when re-entering the quick-tune mode.

\section{DATA ACQUISITION}

Operating the FWCD system becomes a useless endeavor without a data acquisition system to acquire FWCD data synchronized with DIII-D experimental data. Signals emanating from directional couplers, voltage probes, and current probes located at strategic locations along the transmission line network are digitized and stored in local memory by CAMAC instrumentation. The data acquisition program performs a direct memory access (DMA) data transfer and processes the raw data creating a DIII-D shot file. The shot file is then available to the DIII-D "Open System" [4] data acquisition system. Before data can be acquired though, all the GPIB and CAMAC instrumentation must be setup appropriately. Therefore, there are two primary functions of the data acquisition branch of the main program; initialization of the GPIB/CAMAC instrumentation and acquiring data for processing.

Their are nineteen GPIB instruments that can be controlled by the FWCD control console. This poses a small problem 
since the IEEE 488.2 (GPIB) standard limits the protocol to only fourteen devices per GPIB bus. To accommodate the IEEE 488.2 standard, along with device-addressing problems we experienced during program development, we installed three GPIB bus controller cards in the FWCD control console; one controller card for each transmission line system and one for the FWCD control room instrumentation. Of the nineteen GPIB devices nine are CAMAC crates using GPIB crate controllers. These crates are distributed among the three transmission line tuner stations, the DIII-D pit, and the FWCD control room. Since the distances between the GPIB controlled CAMAC crates can span hundreds of feet, violating the wire cable length limitations for interconnecting GPIB devices, fiber optic extender modules are used to allow for transmission of GPIB protocol commands. Therefore, the operator can communicate with all CAMAC instrumentation and either initialize the instrumentation for operation or make instrumentation setup changes as the need arises. Besides the CAMAC instrumentation, there are ten control instruments located in the FWCD control room which are also GPIB controlled. After initializing or changing setups of the GPIB instruments, the status of the instruments is acquired and variables within the main program needed for processing data are defined.

The FWCD main control program and the GPIB instrumentation are synchronized with DIII-D operations via the DIII-D asynchronous and synchronous timing system to ensure that FWCD raw data is meshed properly with the DIII-D data acquisition system. The main program routinely polls for specific DIII-D timing marks and when a specific asynchronous timing signal is received, the FWCD console operator will be alerted that a DIII-D plasma shot is being queued. Once the first synchronous timing signal is received, the main control program will launch the DIII-D shot sequence sub-program. The displayed window on the console displays the FWCD pulse status and any transmission line faults which might have occurred during the rf pulse. After the rf pulse, the digitizers are read followed by execution of the FWCD data archiving program; this status is also displayed on the DIII-D shot sequence window. In order for the raw FWCD data to be processed several variables must be defined so that the FWCD data will be in sync with the rest of the DIII-D world. Digitizer sampling frequency, $\mathrm{rf}$ instrumentation gains, and calibration codes are stored in a labview global routine and are used when constructing the raw data file. The FWCD data archiving program uses the global variables to process the FWCD raw data and creates a DIII-D shot file.

\section{CONCLUSION}

In order for the main program to communicate with the devices within the three primary branches, various intercommunication protocols are supported. A VXI-MXI interface is used to link the FWCD control console to the two rf transmitter control interfaces via two VXI chassis connected to a MXI bus [3]. CAMAC instrumentation is used to monitor DIII-D shot timing sequencing and acquire $\mathrm{rf}$ data. The IEEE 488.2 GPIB protocol is used for data I/O transfer from both CAMAC crates and pulse control instrumentation to the FWCD control console. The control console's Serial port is used to communicate with the transmission line tuning elements. And the console's ethernet port is used to communicate with the DIII-D data acquisition "Open System" architecture [4].

As with any software development endeavor of this magnitude, there are always version upgrades. This paper discussed is what would amount to version one of the DIII-D FWCD control software. Like any other version one software package, there are many needs for improvement. During the past year of DIII-D operations, the FWCD control software was thoroughly tested and areas for improvement documented. Much of the developed improvements were realized as a better understanding of the labview programming environment and of the use of multiple intercommunication protocols were achieved.

\section{REFERENCES}

[1] J.S. deGrassie, et al. "4 MW upgrade of FWCD on DIII-D," in Proc. 15th IEEE/NPSS Symp. on Fusion Engineering, vol. II p. $1073,1993$.

[2] W.P. Cary, et al., "ICH rf system data acquisition and real time control using a microcomputer system," in Proc. of the 15th IEEE/NPSS Symp. on Fusion Engineering, vol. II p. 547, 1993.

[3] National Instruments, "VXI-MXI User Manual," October 1993, p. $1-1$.

[4] P.A. Henline, "Use of open systems for control, analysis, and data acquisition of the DIII-D tokamak," in Proc. of the 15th IEEE/NPSS Symp. on Fusion Engineering, vol. I p. 127, 1993. 